\title{
Video Article \\ Isolation and Culture of Human Mature Adipocytes Using Membrane Mature Adipocyte Aggregate Cultures (MAAC)
}

\author{
Ida Alexandersson ${ }^{1}$, Matthew J. Harms ${ }^{1}$, Jeremie Boucher ${ }^{1,2,3}$ \\ ${ }^{1}$ Bioscience Metabolism, Research and Early Development, Cardiovascular, Renal and Metabolism (CVRM), BioPharmaceuticals R\&D, AstraZeneca \\ ${ }^{2}$ The Lundberg Laboratory for Diabetes Research, University of Gothenburg \\ ${ }^{3}$ Wallenberg Centre for Molecular and Translational Medicine, University of Gothenburg
}

Correspondence to: Jeremie Boucher at jeremie.boucher@astrazeneca.com

URL: https://www.jove.com/video/60485

DOI: doi:10.3791/60485

Keywords: Biology, Issue 156, MAAC, mature adipocyte, culture, transdifferentiation, WAT, BAT, beige, brown, UCP1

Date Published: 2/13/2020

Citation: Alexandersson, I., Harms, M.J., Boucher, J. Isolation and Culture of Human Mature Adipocytes Using Membrane Mature Adipocyte Aggregate Cultures (MAAC). J. Vis. Exp. (156), e60485, doi:10.3791/60485 (2020).

\section{Abstract}

White adipose tissue (WAT) dysregulation plays a central role in development of insulin resistance and type 2 diabetes (T2D). To develop new treatments for T2D, more physiologically relevant in vitro adipocyte models are required. This study describes a new technique to isolate and culture mature human adipocytes. This method is entitled MAAC (membrane mature adipocyte aggregate culture), and compared to other adipocyte in vitro models, MAAC possesses an adipogenic gene signature that is the closest to freshly isolated mature adipocytes. Using MAAC, adipocytes can be cultured from lean and obese patients, different adipose depots, co-cultured with different cell types, and importantly, can be kept in culture for 2 weeks. Functional experiments can also be performed on MAAC including glucose uptake, lipogenesis, and lipolysis. Moreover, MAAC responds robustly to diverse pharmacological agonism and can be used to study adipocyte phenotypic changes, including the transdifferentiation of white adipocytes into brown-like fat cells.

\section{Video Link}

The video component of this article can be found at https://www.jove.com/video/60485/

\section{Introduction}

The worldwide increase in obesity and obesity-related co-morbidities necessitates the development of new therapeutics. White adipose tissue (WAT) is an important regulator of whole-body metabolism, energy homeostasis, and is a central player in the development of insulin resistance and type 2 diabetes (T2D) ${ }^{1,2}$. During chronic excess calorie consumption, adipocytes enlarge to handle the surplus of energy. However, adipocyte lipid storage capacity can become exceeded, resulting in an elevation of circulating levels of fatty acids and increased storage in peripheral non-adipose tissues and leading to lipotoxicity ${ }^{3,4}$.

The lack of adipocyte in vitro models with high translational relevance is a key challenge in the development of new treatments for obesity and T2D. The ex vivo explant model, where small pieces of adipose tissue are cultured, is associated with rapid alterations in adipogenic gene expression driven by hypoxia and inflammation ${ }^{5,6}$. Ceiling cultures (CCs) where mature adipocytes float and adhere to the top of media-filled flasks, rapidly dedifferentiate into fibroblast-like cells lacking lipid ${ }^{7,8,9,10}$. The most commonly used model is adipocytes differentiated in vitro from committed precursors. The differentiated cells are, however, morphologically distinct from mature adipocytes in vivo since they are much smaller in size and lack a unilocular lipid droplet. Other limitations with this model include the unphysiological need of a chemical cocktail to drive differentiation, as well as variability in differentiation efficiency which can be affected by a number of factors ${ }^{11,12,13,14}$.

We have recently developed membrane mature adipocyte aggregate culture (MAAC), a method for long term culture of freshly isolated mature adipocytes, where adipocytes are cultured under permeable membranes ${ }^{10}$. Unbiased analysis of RNA sequencing data has shown that relative to adipose tissue explants and in vitro-differentiated adipocytes, MAAC is most similar to freshly isolated adipocytes. MAAC can be used to culture mature adipocytes isolated from both subcutaneous and visceral adipose tissue, as well as adipocytes from both obese and lean subjects. This methodology permits the study of long-term adipocyte phenotypic changes and facilitates co-culture of adipocytes with other cell types. Here we provide a detailed protocol for the isolation of mature adipocytes from human adipose tissue and how to set up the MAAC system. 


\section{Protocol}

Anonymous samples of adipose tissue were collected from the abdominal region of female patients undergoing elective surgery at Sahlgrenska University Hospital in Gothenburg, Sweden. All study subjects received written and oral information before giving written informed consent for the use of the tissue. The studies were approved by The Regional Ethical Review Board in Gothenburg, Sweden.

NOTE: An overview of the method is provided in Figure 1.

\section{Preparation of Buffers, Tissue Culture Media, and Culture Plates}

1. Prepare a $5 x$ Krebbs Ringer $(\mathrm{KR})$ stock by dissolving $35.1 \mathrm{~g}$ of $\mathrm{NaCl}, 1.75 \mathrm{~g}$ of $\mathrm{KCl}, 0.82 \mathrm{~g}$ of $\mathrm{KH}_{2} \mathrm{PO}_{4}$, and $1.48 \mathrm{~g}$ of $\mathrm{MgSO}_{4} \cdot 7 \mathrm{H}_{2} \mathrm{O}$ in $900 \mathrm{~mL}$ of water. Add $1.84 \mathrm{~g}$ of $\mathrm{CaCl}_{2} \cdot 2 \mathrm{H}_{2} \mathrm{O}$ and adjust the volume to $1 \mathrm{~L}$ by adding water. Sterile filter through a $0.22 \mu \mathrm{m}$ filter and store at $4{ }^{\circ} \mathrm{C}$.

2. From the $5 x \mathrm{KR}$ stock, prepare $1 \mathrm{~L}$ of buffer containing $1 \times \mathrm{KR}, 25 \mathrm{mM}$ HEPES, $2 \mathrm{mM}$ glucose, and $2 \%$ bovine serum albumin (BSA) (referred to hereafter as wash buffer). Adjust the $\mathrm{pH}$ to 7.4 .

3. Prepare $500 \mathrm{~mL}$ of collagenase buffer containing $1 \times \mathrm{HBSS}+\mathrm{CaCl}_{2}+\mathrm{MgCl}_{2}, 2 \% \mathrm{BSA}$, and $450 \mathrm{~mL}$ of water (referred to hereafter as digestion buffer).

NOTE: The collagenase should not be added to the digestion buffer until after the adipose tissue has been weighed in step 2.2 .

4. Adjust the $\mathrm{pH}$ of Medium 199 to 7.4 .

5. Sterile filter both buffers and medium 199 through a $0.22 \mu \mathrm{m}$ filter flask and warm to $37^{\circ} \mathrm{C}$.

NOTE: To save time, the tissue culture media can be prepared and added to plates before processing the adipose tissue. For a 24-well plate format (Figure 1A), use 0.5-1 mL/well of Dulbecco's modified Eagle's medium/Ham's nutrient mixture F12 (DMEM/F12), 10\% fetal bovine serum (FBS), 1\% penicillin-streptomycin (penn/strep) and $20 \mathrm{nM}$ insulin. Small molecules and other stable pharmacological agents can also be added at this time to the media in the desired layout. Place the plates in a tissue culture incubator $\left(37^{\circ} \mathrm{C}, 5 \% \mathrm{CO}_{2}\right)$.

\section{Dissection of Human Subcutaneous Adipose Tissue}

NOTE: Work within a biological safety cabinet and use sterile technique throughout the isolation process, as well as exclusive use of autoclaved and sterile equipment.

1. Place the human adipose tissue in a $15 \mathrm{~cm}$ petri dish and add a small volume of medium 199 to keep it moisturized during the dissection.

2. Working with pieces of adipose approximately the size of a golf ball, grasp large fibrotic vessels with tweezers and gently release the adipocytes by scraping the adipose with the back of closed scissors. Discard the large pieces of fibrotic tissue. Weigh the trimmed fat.

\section{Collagenase Treatment}

1. Add collagenase to the digestion buffer (see step 1.3) at a concentration of $1 \mathrm{mg} / \mathrm{mL}$. Sterile filter the solution using a $0.22 \mu \mathrm{m}$ sterile filter. NOTE: Three $\mathrm{mL}$ of digestion buffer per gram of fat is recommended (i.e., for $100 \mathrm{~g}$ of fat, prepare $300 \mathrm{~mL}$ of digestion buffer with $300 \mathrm{mg}$ of type 2 collagenase).

CAUTION: Type 2 Collagenase is hazardous to eyes, skin and may cause respiratory irritation. Wear gloves, eye protection, and work in a ventilated hood when handling the collagenase.

2. Move approximately $10 \mathrm{~g}$ of adipose tissue to a $15 \mathrm{~cm}$ petri dish and mince the fat carefully using a pair of curved scissors until it becomes a smooth homogenous mixture and there are no large pieces of adipose left. Repeat the process until all of the fat has been processed. NOTE: Each round should take approximately $2 \mathrm{~min}$ and the adipose pieces should be small enough so that they can be pipetted using a wide-bore pipette tip. This step is crucial to yielding high quality adipocytes. If the pieces are too large, digestion times will have to be extended, compromising cell viabilty.

3. Transfer the minced fat into $50 \mathrm{~mL}$ conical tubes using a spoon. Add $10 \mathrm{~mL}$ of minced tissue and $30 \mathrm{~mL}$ of digestion buffer to each tube. Scale down to appropriate volumes if less than $10 \mathrm{~mL}$ of minced fat is available.

4. Digest the tissue at $37^{\circ} \mathrm{C}$ in a shaking incubator with constant agitation at $150 \mathrm{rpm}$ for $30-45 \mathrm{~min}$. After 30 min, check on the process every 5 min to avoid over-digestion.

NOTE: The digestion is complete when the adipose solution is homogenous without any large pieces and has an apricot color.

\section{Filtration of the Cell Suspension and Purification of the Mature Adipocytes}

1. Place a funnel on top of a $1 \mathrm{~L}$ sterile flask and place a sterile $250 \mu \mathrm{m}$ mesh filter inside the funnel. Pour the digested fat solution into the filter to remove the undigested tissue.

2. When all of the adipocyte suspension has passed through the filter, gently squeeze the mesh filter to increase the yield of adipocytes. Pour approximately $50-100 \mathrm{~mL}$ of wash buffer into the filter to rinse it and squeeze the filter again.

3. Pour the isolated adipocyte suspension from the flask into a separation funnel and add wash buffer until the funnel is almost completely filled. Gently invert the funnel a few times to mix the adipocyte suspension with the buffer.

4. Let the suspension stand for 2-3 min until there is a distinct separation of 2 layers (Figure 1B), with a top yellow layer containing the mature adipocytes and free lipid and a bottom layer containing the buffer and the adipose stroma vascular fraction (SVF).

5. Open the nozzle on the separation funnel, and slowly elute the bottom solution into a sterile flask (cells from the SVF can be pelleted and collected after centrifugation at $200 \times \mathrm{g}$ for $7 \mathrm{~min}$ ). Keep the top layer with the mature adipocytes in the separation funnel.

6. Repeat the washing process in steps $4.3-4.5$ three times to thoroughly wash the mature adipocytes and remove all of the collagenase. 


\section{Packing of the Mature Adipocytes}

1. Open the nozzle and collect the purified mature adipocyte suspension into $50 \mathrm{~mL}$ conical tubes.

2. Lightly pack the mature adipocytes by spinning the tubes at $50 \times \mathrm{g}$ for $3 \mathrm{~min}$.

3. Use a $18 \mathrm{G}$ needle and a syringe to remove the remaining wash buffer below the adipocyte suspension.

4. Remove the free lipid layer (oil from the small portion of adipocytes that broke during the isolation procedure) floating on top of the mature adipocytes by using a pipette.

NOTE: To reduce the risk that the adipocytes will drip off the membranes in step 6.5, it is important that the lipid layer and all wash buffer have been removed when the mature adipocytes are seeded onto membranes. For this reason collect the adipocytes in 3 tubes. The samples that are collected last will have the most carryover lipid and will therefore be of the lowest quality. However, with careful pipetting these samples can be used.

\section{Seeding of Mature Adipocytes}

1. Open the package containing the permeable membrane inserts (Table of Materials) and take out the membrane component. Flip it upside down and place on a sterile surface so the membranes face the ceiling.

2. Pipette $30 \mu \mathrm{L}$ of packed mature adipocytes onto each of the membranes (Figure 1C). Avoid touching the membrane with the tip. Use widebore pipette tips to seed the cells, or use scissors to cut off a small piece of a pipette tip to make it wider.

3. Gently invert the $50 \mathrm{~mL}$ tubes with packed adipocytes several times throughout the seeding process to ensure an even distribution of adipocytes with different sizes.

4. Bring the prepared multiwell plates containing the media from the incubator to the biosafety cabinet and remove the lids. Pick up the membranes seeded with adipocytes and grasp it from the bottom so that it can be inverted in step 6.5.

5. In one smooth movement invert the membranes with the adipocytes on top so that the seeded adipocytes are now facing down (Figure 1D) Lower the plate with adipocytes into the wells containing media (Figure 1E).

6. Put the lid on the plate and carefully transfer the plate into a tissue culture incubator. Avoid rapid movement of the plate since adipocytes can easily be dislodged initially.

NOTE: It takes a few days for the cells to adhere more firmly to the membrane.

\section{Maintenance of Adipocytes and Harvesting for Analysis}

1. Change the media at least every 7 days. Remove and add media via the cutout hole in each membrane insert. To remove the media, use a syringe and a needle, an aspirating wand, or a pipette with a p200 tip. To add media, use a pipette and slowly pipette new media into the wells along the side of the wall to avoid disturbing the adipocytes.

NOTE: Adipocytes have been cultured for 2 weeks with one media change after 7 days. However, different adipose origins and experimental questions may benefit from increased media changes.

2. To harvest RNA, remove the media as described in step 7.1, and add $500 \mu \mathrm{L}$ of lysis buffer (Table of Materials) directly into the wells to lyse the cells. To fix the cells for imaging, add formaldehyde directly to the well at a final concentration of $1 \%$.

NOTE: The cells can then be stored at $4{ }^{\circ} \mathrm{C}$.

\section{Representative Results}

Mature adipocytes cultured as MAAC preserves their function, phenotype, and can be used to study adipocyte responses to various pharmacological treatments. After 1 week of culture MAAC isolated from subcutaneous adipose tissue maintain the characteristic unilocular lipid droplet found only in mature adipocytes (Figure 2A). MAAC was cultured for 1 week while treated with either the PPARy agonists rosiglitazone (Rosi) and pioglitazone (Pio), or the glucocorticoid receptor (GR) agonist dexamethasone (Dex) to determine if different nuclear hormone receptor (NHR) agonists drive predicted changes in downstream target genes in MAAC. Rosi and Pio increased the expression of the PPARy responsive genes FABP4 and $L P L$ by 4 and 2-3 fold, respectively, whereas dexamethasone had no effect (Figure 2B). Similarly, dexamethasone robustly drove the gene expression of the GR target genes $A P O D$ and FKBP5 by 13- and 55-fold respectively, while the PPARY agonists had no significant effects. We have previously demonstrated that freshly isolated human mature white adipocytes can transdifferentiate into a brown-like phenotype in MAAC when treated with Rosi ${ }^{10}$. A 7 day treatment with Rosi or Pio robustly induced the gene expression of the brown fat-specific gene UCP1 by 44-65 fold, as well as increased the expression of the brown fat marker PDK4 12-18 fold (Figure 2C). 
A. Set up experimental plates

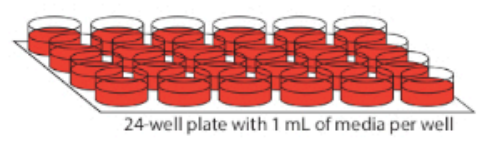

B. Isolate adipocytes
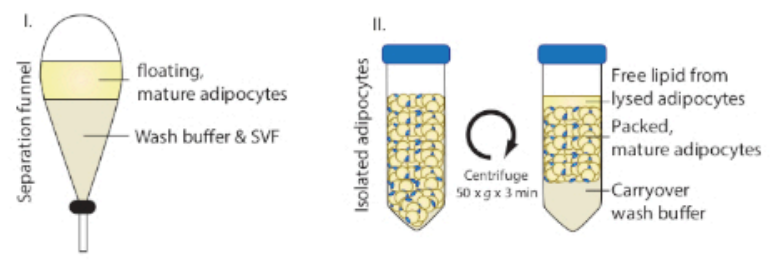

C. Plate the mature adipocytes

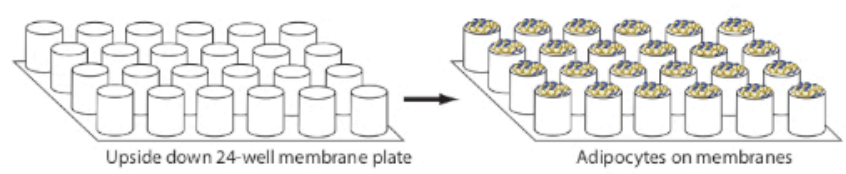

D. Invert the membranes with adipocytes attached

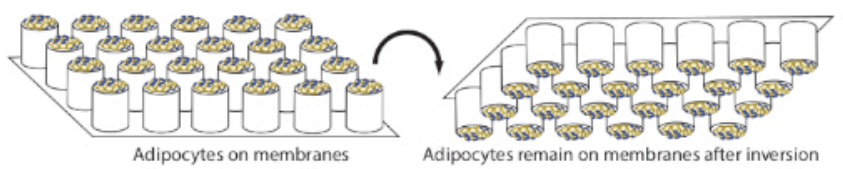

E. Lower the adipocytes into the medium

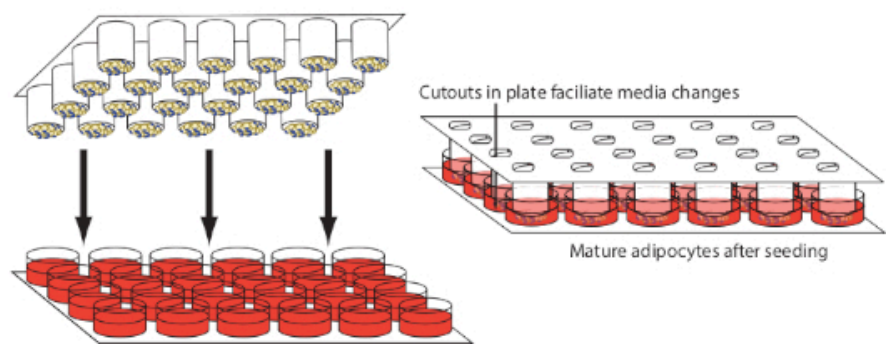

Figure 1: Visual diagram of MAAC setup. (A) Preparation of medium. (B) Isolation and packing of mature adipocytes. (C) Seeding mature adipocytes onto membranes. (D) Inverting membranes while keeping adipocytes attached. (E) Lowering the membranes into the medium and changing the medium. This figure has been modified from Harms et al. ${ }^{10}$. Please click here to view a larger version of this figure. 
A
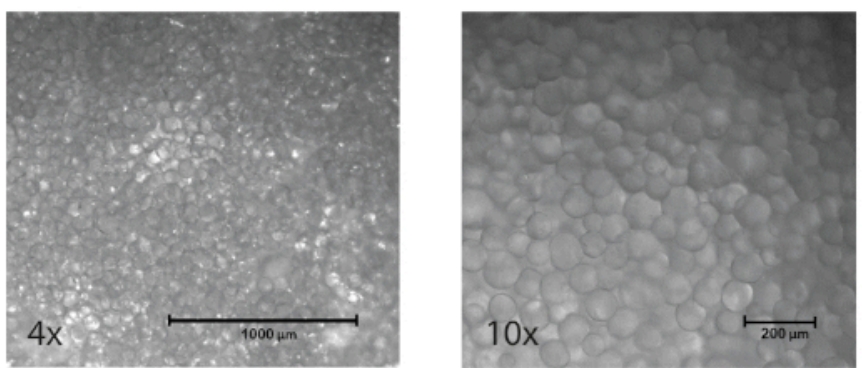

B

PPARY responsive genes

FABP4

$L P L$

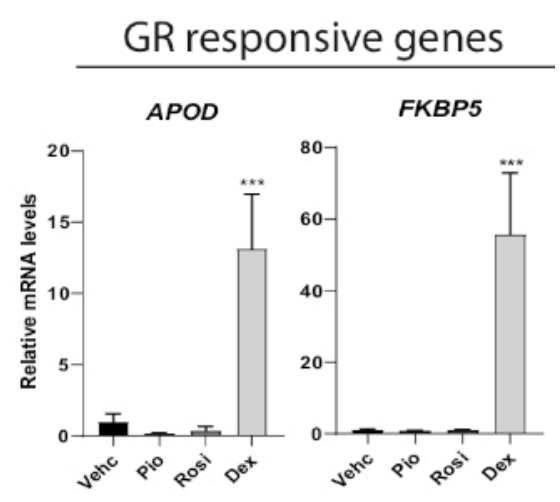

C

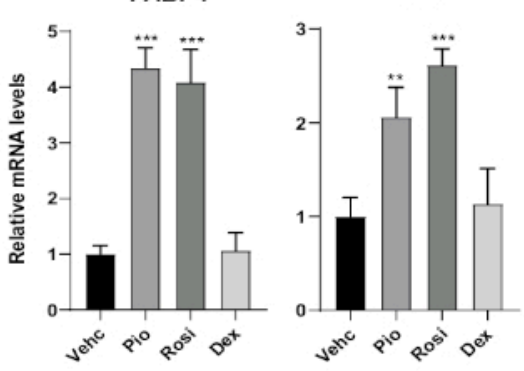

\section{Brown fat-enriched genes}

UCP1 PDK4
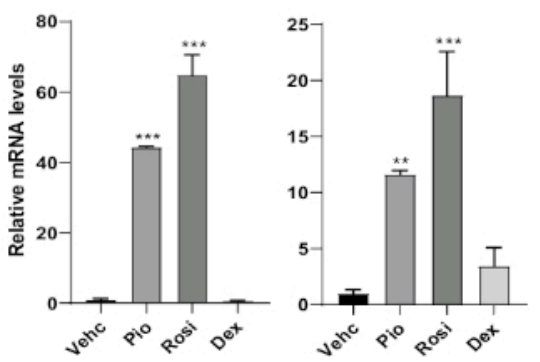

Figure 2: MAAC maintains unilocular appearance through one week and respond to diverse pharmacological agonism. (A)

Representative $4 x$ and 10x bright field images of MAAC after one week of culture. Adipocytes that have an average diameter of $100 \mu \mathrm{m}$ with large unilocular lipid droplets are easily discernable. (B) mRNA levels of PPARy target genes and glucocorticoid receptor (GR) target genes in MAAC after 7 days of treatment with vehicle (Vehc), rosiglitazone (Rosi), pioglitazone (Pio), or dexamethasone (Dex). Rosi, Pio and Dex were all used at a final concentration of $10 \mu \mathrm{M}$. (C) mRNA levels of brown fat-enriched genes in MAAC after 7 days of treatment with vehicle (Vehc), rosiglitazone (Rosi), pioglitazone (Pio), or dexamethasone (Dex). Rosi, Pio and Dex were all used at a final concentration of $10 \mu \mathrm{M}$. For all gene expression data, TATA-binding protein (TBP) was used as an internal normalization control. Statistics were calculated using one-way ANOVA with Tukey's multiple comparisons test. (mean $\pm \mathrm{SD}, \mathrm{n}=3,{ }^{*} \mathrm{p}<0.05$; ${ }^{* *} \mathrm{p}<0.01$; ${ }^{* \star *} \mathrm{p}<0.001$ ). Please click here to view a larger version of this figure. 


\section{Discussion}

Membrane mature adipocyte aggregate culture (MAAC) is a new method for the long-term culture of freshly isolated mature adipocytes. In setting up MAAC there are a few critical steps in the protocol that greatly impact the yield, quality, and viability of the mature adipocytes. Much effort should be put into mincing the fat in step 3.2 since this step directly influences the amount of time the adipocytes are exposed to the collagenase. If the pieces of adipose are too large, the digestion time will have to be extended which negatively impacts the viability of the cells Conversely, if the tissue is processed too finely with scissors, viability can be impacted as well. For successful culture of mature adipocytes as MAAC, one should give special attention to the following steps: for successful seeding of the adipocytes onto the membranes, it is crucial that free lipid and carryover wash buffer is removed from the mature adipocytes in steps 5.3 and 5.4. Remaining lipid or wash buffer will reduce the surface tension of the adipocytes and increase the risk of adipocytes dripping-off the membranes when they are flipped. When the adipocytes are seeded and in media, the cells remain in contact with the membrane primarily through buoyancy, thus a slow and gentle technique is recommended when changing media to not lose cells. Remove media from the bottom of the wells as described in step 7.1 and add media by slowly pipetting media down the sides of the walls. Lastly, a time saving suggestion is to prepare the plates with media and treatments before the adipocyte isolation process. Particularly for complex experimental designs, pre-preparing the plates can save much time and ensures that the adipocytes are placed in media with their treatments as soon as they are isolated.

One benefit of using MAAC compared to differentiating preadipocytes is that the MAAC media used is very simple and does not require an unphysiological hormone cocktail. Here we have cultured MAAC in glucose-rich media (DMEM/F12), 10\% FBS, $1 \%$ penn/strep, and $20 \mathrm{nM}$ insulin. Importantly, we have found that insulin is absolutely required for the rosiglitazone/pioglitazone driven induction of $U C P 1^{10}$. Insulin, however, is not required to maintain the cells' adipogenic phenotype. Thus, depending upon the experimental question, insulin can be included or omitted.

The procedure detailed above has been optimized for the isolation and culture of human adipocytes. However, mouse, and possibly other organism's adipocytes, can also be cultured as MAAC. If mouse adipocytes are to be cultured as MAAC there are additional considerations and precautions that should be kept in mind. We have found that mature adipocytes from mice are much more fragile than those from humans. As a result, the digestion time should be shortened to an absolute minimum to increase cell viability. We also found that adipocytes from young mice (8-week-old and younger) provided the most robust and reproducible results. Lastly, mouse MAAC can be cultured for up to one week, however given that their adipogenic phenotype appears less stable than humans (which can be cultured through at least two weeks) we recommend culturing mouse MAAC for the minimum required time to address experimental questions.

Since the MAAC model is based on using permeable membranes, one advantage of this technique is the possibility of co-culturing mature adipocytes with other cell types. We have previously demonstrated the ability of mature adipocytes and macrophages to crosstalk through the use of MAAC ${ }^{10}$. This opens up opportunities to further explore the linkage between obesity, insulin resistance, and immune responses ${ }^{15,16,17}$. Future experiments could incorporate other cell types such as hepatocytes, preadipocytes, endothelial cells, or pancreatic cells to further increase the complexity and the translational relevance of the MAAC model and investigate crosstalk between multiple cell types.

Even though MAAC has been shown to be superior at maintaining functionality and identity of the adipocytes relative to other adipocyte in vitro models, it also has limitations that need to be considered. In comparison to using adipocytes differentiated from precursor cells, MAAC is a more laborious and time-consuming model. Plates with membranes are also more expensive compared to regular cell culture plates. Importantly, mature adipocytes need to be freshly isolated each time upon seeding and cannot be expanded or frozen into stocks like precursor cells. Thus, this requires having access to fresh white adipose tissue samples, but also adds a level of complexity stemming from donor to donor variations.

Here we have presented a detailed protocol for isolating human mature adipocytes and setting up MAAC. We have demonstrated that adipocytes cultured as MAAC remains viable through two weeks, their adipogenic gene signature is preserved, and they respond to diverse pharmacological agonism. Using MAAC allows for the study of cross-talk betweeen adipocytes and other cell types, and the assessment of long term phenotypic changes of mature adipocytes in response to different stimuli.

\section{Disclosures}

The authors have nothing to disclose.

\section{Acknowledgments}

We thank Xiao-Rong Peng and Stefan Hallen for providing resources and optimizing the adipocyte isolation, Martin Uhrbom for technical assistance, and Daniel Olausson and Malin Lönn for coordinating and providing the human adipose.

\section{References}

1. Guilherme, A., Virbasius, J. V., Puri, V., Czech, M. P. Adipocyte dysfunctions linking obesity to insulin resistance and type 2 diabetes. Nature Reviews Molecular Cell Biology. 9 (5), 367-377 (2008).

2. Rosen, E. D., Spiegelman, B. M. What we talk about when we talk about fat. Cell. 156 (1-2), $20-44$ (2014).

3. Lotta, L. A. et al. Integrative genomic analysis implicates limited peripheral adipose storage capacity in the pathogenesis of human insulin resistance. Nature Genetics. 49 (1), 17-26 (2017).

4. Gustafson, B., Hedjazifar, S., Gogg, S., Hammarstedt, A., Smith, U. Insulin resistance and impaired adipogenesis. Trends in Endocrinology and Metabolism. 26 (4), 193-200 (2015). 
5. Gesta, S. et al. Culture of human adipose tissue explants leads to profound alteration of adipocyte gene expression. Hormone and Metabolic Research. 35 (3), 158-163 (2003).

6. Fain, J. N., Cheema, P., Madan, A. K., Tichansky, D. S. Dexamethasone and the inflammatory response in explants of human omental adipose tissue. Molecular and Cellular Endocrinology. 315 (1-2), 292-298 (2010).

7. Lessard, J. et al. Characterization of dedifferentiating human mature adipocytes from the visceral and subcutaneous fat compartments: fibroblast-activation protein alpha and dipeptidyl peptidase 4 as major components of matrix remodeling. PLoS One. 10 (3), e0122065 (2015).

8. Asada, S. et al. Ceiling culture-derived proliferative adipocytes retain high adipogenic potential suitable for use as a vehicle for gene transduction therapy. American Journal of Physiology - Cell Physiology. 301 (1), C181-185 (2011).

9. Shen, J. F., Sugawara, A., Yamashita, J., Ogura, H., Sato, S. Dedifferentiated fat cells: an alternative source of adult multipotent cells from the adipose tissues. International Journal of Oral Science. 3 (3), 117-124 (2011).

10. Harms, M. J. et al. Mature Human White Adipocytes Cultured under Membranes Maintain Identity, Function, and Can Transdifferentiate into Brown-like Adipocytes. Cell Reports. 27 (1), 213-225.e215 (2019).

11. Wang, Q. A., Scherer, P. E., Gupta, R. K. Improved methodologies for the study of adipose biology: insights gained and opportunities ahead. Journal of Lipid Research. 55 (4), 605-624 (2014).

12. Gregoire, F. M., Smas, C. M., Sul, H. S. Understanding adipocyte differentiation. Physiological Reviews. 78 (3), $783-809$ (1998).

13. Ruiz-Ojeda, F. J., Ruperez, A. I., Gomez-Llorente, C., Gil, A., Aguilera, C. M. Cell Models and Their Application for Studying Adipogenic Differentiation in Relation to Obesity: A Review. International Journal of Molecular Sciences. 17 (7), 1040 (2016).

14. McBeath, R., Pirone, D. M., Nelson, C. M., Bhadriraju, K., Chen, C. S. Cell shape, cytoskeletal tension, and RhoA regulate stem cell lineage commitment. Developmental Cell. 6 (4), 483-495 (2004).

15. Lackey, D. E., Olefsky, J. M. Regulation of metabolism by the innate immune system. Nature Reviews Endocrinology. 12 (1), 15-28 (2016)

16. Saltiel, A. R., Olefsky, J. M. Inflammatory mechanisms linking obesity and metabolic disease. The Journal of clinical investigation. 127 (1), 1-4 (2017).

17. Lee, Y. S., Wollam, J., Olefsky, J. M. An Integrated View of Immunometabolism. Cell. 172 (1-2), 22-40 (2018). 